Type of the Paper (Proceedings, Abstract, Extended Abstract, Editorial, etc.)

\title{
Inflation, primordial black holes and induced gravitational waves from modified supergravity
}

\author{
Sergei V. Ketov
}

Citation: Lastname, F.; Lastname, F.; Lastname, F. Title. Proceedings 2021, 68, x. https://doi.org/10.3390/xxxxx

Published: date

Publisher's Note: MDPI stays neutral with regard to jurisdictional claims in published maps and institutional affiliations.

Copyright: (c) 2021 by the authors. Submitted for possible open access publication under the terms and conditions of the Creative Commons Attribution (CC BY) license (http://creativecommons.org/licenses /by/4.0/).
1 Affiliation 1; e-mail@e-mail.com

\begin{abstract}
Modified (Starobinsky type) supergravity is used for a viable (unified) description of cosmological inflation and formation of primordial black holes and dark matter in the early Universe. A specific class of models is proposed and investigated in detail. Their observational predictions for primordial black hole masses, dark matter and induced gravitational waves are derived and compared to the current and future astrophysical and cosmological observations. Our approach naturally leads to the two-scalar-field attractor-type double inflation, whose first stage is driven by Starobinsky scalaron and whose second stage is driven by another scalar field which belongs to a supergravity multiplet. The scalar potential and the kinetic terms are derived, the vacua are studied, and the inflationary dynamics of those two scalars is investigated. We numerically compute the power spectra and find the ultra-slow-roll regime leading to an enhancement (peak) in the scalar power spectrum. This leads to an efficient formation of primordial black holes. We estimate their masses and find their density fraction as part of dark matter. We show that our modified supergravity models are in agreement with inflationary observables, while they predict the primordial black holes masses in the range between $10^{\wedge} 16 \mathrm{~g}$ and $10^{\wedge} 20 \mathrm{~g}$. In this sense, modified supergravity provides a natural top-down approach for explaining and unifying the origin of inflation and the dark matter in the form of primordial black holes.
\end{abstract}

Keywords: inflation, primordial black holes, dark matter, supergravity

Author Contributions: For research articles with several authors, a short paragraph specifying their individual contributions must be provided. The following statements should be used "Conceptualization, X.X. and Y.Y.; methodology, X.X.; software, X.X.; validation, X.X., Y.Y. and Z.Z.; formal analysis, X.X.; investigation, X.X.; resources, X.X.; data curation, $X . X . ;$ writing-original draft preparation, X.X.; writing - review and editing, X.X.; visualization, X.X.; supervision, X.X.; project administration, X.X.; funding acquisition, Y.Y. All authors have read and agreed to the published version of the manuscript." Please turn to the CRediT taxonomy for the term explanation. Authorship must be limited to those who have contributed substantially to the work reported.

Funding: Please add: "This research received no external funding" or "This research was funded by NAME OF FUNDER, grant number XXX" and "The APC was funded by XXX". Check carefully that the details given are accurate and use the standard spelling of funding agency names at https://search.crossref.org/funding. Any errors may affect your future funding.

Acknowledgments: In this section, you can acknowledge any support given which is not covered by the author contribution or funding sections. This may include administrative and technical support, or donations in kind (e.g., materials used for experiments).

Conflicts of Interest: Declare conflicts of interest or state "The authors declare no conflict of interest." Authors must identify and declare any personal circumstances or interest that may be perceived as inappropriately influencing the representation or interpretation of reported research results. Any role of the funders in the design of the study; in the collection, analyses or interpretation of data; in the writing of the manuscript, or in the decision to publish the results must be declared in this section. If there is no role, please state "The funders had no role in the design of the study; in 
the collection, analyses, or interpretation of data; in the writing of the manuscript, or in the decision to publish the results". 


\section{Appendix A}

The appendix is an optional section that can contain details and data supplemental to the main text-for example, explanations of experimental details that would disrupt the flow of the main text but nonetheless remain crucial to understanding and reproducing the research shown; figures of replicates for experiments of which representative data is shown in the main text can be added here if brief, or as Supplementary data. Mathematical proofs of results not central to the paper can be added as an appendix.

\section{Appendix B}

All appendix sections must be cited in the main text. In the appendices, Figures, Tables, etc. should be labeled starting with "A" - e.g., Figure A1, Figure A2, etc.

\section{References}

References must be numbered in order of appearance in the text (including citations in tables and legends) and listed individually at the end of the manuscript. We recommend preparing the references with a bibliography software package, such as EndNote, ReferenceManager or Zotero to avoid typing mistakes and duplicated references. Include the digital object identifier (DOI) for all references where available.

Citations and references in the Supplementary Materials are permitted provided that they also appear in the reference list here.

In the text, reference numbers should be placed in square brackets [ ] and placed before the punctuation; for example [1], [1-3] or [1,3]. For embedded citations in the text with pagination, use both parentheses and brackets to indicate the reference number and page numbers; for example [5] (p. 10), or [6] (pp. 101-105).

1. Author 1, A.B.; Author 2, C.D. Title of the article. Abbreviated Journal Name Year, Volume, page range.

2. Author 1, A.; Author 2, B. Title of the chapter. In Book Title, 2nd ed.; Editor 1, A., Editor 2, B., Eds.; Publisher: Publisher Location, Country, 2007; Volume 3, pp. 154-196.

3. Author 1, A.; Author 2, B. Book Title, 3rd ed.; Publisher: Publisher Location, Country, 2008; pp. 154-196.

4. Author 1, A.B.; Author 2, C. Title of Unpublished Work. Abbreviated Journal Name stage of publication (under review; accepted; in press).

5. Author 1, A.B. (University, City, State, Country); Author 2, C. (Institute, City, State, Country). Personal communication, 2012.

6. Author 1, A.B.; Author 2, C.D.; Author 3, E.F. Title of Presentation. In Title of the Collected Work (if available), Proceedings of the Name of the Conference, Location of Conference, Country, Date of Conference; Editor 1, Editor 2, Eds. (if available); Publisher: City, Country, Year (if available); Abstract Number (optional), Pagination (optional).

7. Author 1, A.B. Title of Thesis. Level of Thesis, Degree-Granting University, Location of University, Date of Completion.

8. Title of Site. Available online: URL (accessed on Day Month Year). 